\title{
Immunological relatedness of the LamB proteins among members of Enterobacteriaceae
}

\author{
Catherine Werts, David O’Callaghan, Maurice Hofnung and Alain Charbit* \\ Unité de Programmation Moléculaire et Toxicologie Génétique, CNRS URA1444, Institut Pasteur, \\ 25 rue du Dr Roux, 75724 Paris Cedex, France
}

(Received 15 September 1992; revised 18 November 1992; accepted 27 November 1992)

\begin{abstract}
We have studied the immunological relatedness of LamB proteins from a wide range of enterobacterial species, using antibodies directed against denatured Escherichia coli K12 and Klebsiella pneumoniae LamB proteins (LamBE.c. and LamBK.p., respectively), and anti-peptide antibodies directed against 10 distinct loops of LamB from $E$. coli K12 predicted to protrude either side of the outer membrane. We have shown that a protein immunologically related to LamBE.c. and LamBK.p. was present in all members of Enterobacteriaceae tested. A protein recognized by several anti-peptide antibodies was identified in E. coli, Shigella sonnei, Salmonella typhimurium and Kleb. pneumoniae, as well as in two Citrobacter species, two Enterobacter species and Kluyvera ascorbata. The recognition patterns obtained with the anti-peptide antibodies were in agreement with the LamB protein sequence data available. They indicated that the cell surface and also the periplasmic loops of LamB are subject to great antigenic variability.
\end{abstract}

\section{Introduction}

In Escherichia coli $\mathrm{K} 12$, the lamB gene encodes the maltose-inducible integral outer-membrane protein LamB. LamB constitutes a pore specifically involved in the diffusion of maltose and maltodextrins (for reviews, see Hengge \& Boos, 1983; Naikaido \& Vaara, 1985). It also serves as a cell-surface receptor for a number of phages, including $\lambda$ (Charbit $\&$ Hofnung, 1985). The presence of a protein immunologically related to the $E$. coli LamB protein has been reported in several members of the family Enterobacteriaceae (Pick \& Wober, 1979; Bloch \& Desaymard, 1985). Comparison of the antigenic determinants of LamB from different enterobacteria, using monoclonal antibodies (mAb) directed against surface-exposed or periplasmic portions of the $E$. coli LamB protein (LamBE.c.), indicated great variability of the external epitopes, and better conservation of the internal ones. Similar studies using mAbs or anti-peptide antibodies have been carried out on other outermembrane proteins such as FepA (Rutz et al., 1991) and OmpC, OmpD (Singh et al., 1992) and $\mathrm{P} 2$ porins (Hamel et al., 1992). They allowed the identification of both

\footnotetext{
*Author for correspondence. Tel. 1456888 30; fax 145688834 .
}

conserved and highly variable epitopes, providing information on the structural organization of these proteins in the outer membrane.

The $\operatorname{lam} B$ genes from four enterobacteria have now been sequenced: E. coli (Clément \& Hofnung, 1981), Shigella sonnei (Roessner \& Ihler, 1987), Salmonella typhimurium (Francoz et al., 1990), and Klebsiella pneumoniae (Werts et al., 1992). Comparison of their deduced amino acid sequences revealed an overall high degree of similarity. The data showed that the $\mathrm{N}$ terminal third of LamB was the most conserved part of the molecule and that the variability occurred mainly within surface-exposed regions of the protein (Charbit $e t$ al., 1988; Werts et al., 1992).

In the present work, we have studied the immunological relatedness of the LamB proteins from 15 enterobacterial species (Table 1), with either polyclonal antibodies directed against denatured $E$. coli LamB and Kleb. pneumoniae LamB (lamBK.p.), or anti-peptide antibodies directed against 10 different portions of the LamBE.c. protein scattered along the polypeptide chain. Four peptide sequences correspond to regions predicted to be facing the periplasm, and the six others to regions exposed at the cell surface (Fig. 1a). The 15 species chosen were all able to utilize maltose as carbon source. Two of them were unable to use dextrins as carbon 
source. They represent a large panel of Enterobacteriaceae, all more or less related to E. coli.

\section{Methods}

Strains, media, growth on dextrins and phage sensitivity patterns. The strains used in this study are listed in Table 1.The ability of the different enterobacteria to utilize maltose and maltodextrins as carbon source was tested at $30^{\circ} \mathrm{C}$ on MacConkey agar supplemented with maltose $(0.5 \%)$ or dextrins $(0.5 \%)$. On these media, bacteria able to utilize the sugar as carbon source form dark red colonies $\left(\mathrm{Mal}^{+}\right.$or $\mathrm{Dex}^{+}$ phenotype), whereas those which are unable to do so form pink or white colonies $\left(\mathrm{Mal}^{+}\right.$or Dex ${ }^{-}$phenotype). All the strains were tested for their in vivo sensitivity to phage $\lambda$, as well as to 11 other LamBE.c.specific phages by the spot-test procedure (Charbit \& Hofnung, 1985).

Immunization procedures and antibodies. Two polyclonal antibodies raised against denatured (monomeric) LamB protein were used: (i) a rabbit antibody directed against the denatured LamB protein from $E$. coli K12 (called here anti-LamBE.c. antibody; Boulain et al., 1986) and (ii) a rabbit antibody directed against the denatured $\mathrm{LamB}$ protein from Kleb. pneumoniae (anti-LamBK.p. antibody). This serum was obtained after intradermal immunization of rabbits with $50 \mu \mathrm{g}$ denatured LamBK.p. The first injection was performed in Freund's complete adjuvant (FCA) with denatured LamBK.p. obtained after electroelution of the monomeric form of the protein from a $10 \%(w / v)$ SDS-polyacrylamide gel. Two booster injections were performed at 29 and 88 days with $50 \mu \mathrm{g}$ of the affinity-purified (Werts et al., 1992) heatdenatured protein in Freund's incomplete adjuvant (FIA).

Ten polyclonal anti-LamBE.c. peptide antibodies were used in this study. For simplification, all the antibodies directed against cellsurface-predicted regions of LamB will be preceded by $\mathrm{E}$ (for external), and those directed against regions facing the periplasm by I (for internal). Four of these anti-peptide antibodies (anti-I 1, anti-E 2, antiE 3 and anti-E 4) have been described previously (Molla et al., 1989). Anti-peptide I 1 was able to react with native purified LamBE.c., but not on the surface of intact bacteria, whereas anti-E 2, E 3 and E 4 were able to react with LamBE.c. at the surface of intact cells. Six new anti-

\section{Table 1. Enterobacterial strains used in this study}

All strains come from the Service des Entérobactéries (Pasteur Institute) except $E$. coli strains (laboratory collection), Kleb. pneumoniae KAY 2026 (Springer \& Lengeler) and Ent. cloacae which is a clinical isolate from the Villeneuve-Saint-Georges Hospital, France (A. Dublanchet).

\begin{tabular}{ll}
\hline \multicolumn{1}{c}{ Species } & \multicolumn{1}{c}{ Strain } \\
\hline Escherichia coli & P4X8 $\left(\mathrm{LamB}^{+}\right)$ \\
Escherichia coli & pop6510 $\left(\mathrm{LamB}^{-}\right)$ \\
Shigella sonnei & S60-80 \\
Salmonella typhimurium & LT2 \\
Citrobacter freundii & CDC 22-76 \\
Citrobacter diversus & CDC 1066-71 \\
Klebsiella pneumoniae & KAY 2026 \\
Enterobacter cloacae & \\
Enterobacter aerogenes & A1 \\
Erwinia rhapontici & 1025 \\
Erwinia herbicola & E20 \\
Hafnia alvei & I 563272 \\
Serratia marcescens & T 504 (ATCC 13880) \\
Edwardsiella tarda & T 10396 \\
Kluyvera ascorbata & T 648-74 \\
Providencia heimbachae & T 8025-83 \\
\hline \hline
\end{tabular}

LamBE.c. peptide antibodies (see location on the LamBE.c. folding model, Fig. 1) were obtained: anti-I 12, anti-I 15, anti-I 16, and anti-E 5, anti-E 7 and anti-E 13. Peptides were synthesized according to Merrifield (1963) and were coupled to ovalbumin using $1 \%(\mathrm{v} / \mathrm{v})$ glutaraldehyde (Harlow \& Lane, 1988). For each peptide, three female $\mathrm{BALB} / \mathrm{c}$ mice were immunized subcutaneously with $100 \mu \mathrm{g}$ peptide conjugate, in FCA. Two subsequent injections were done intraperitoneally with $100 \mu \mathrm{g}$ peptide conjugate in FIA at 21 and 50 days. Blood samples were taken at 30 and 59 days. Animals were sacrificed on day 66.

(a)

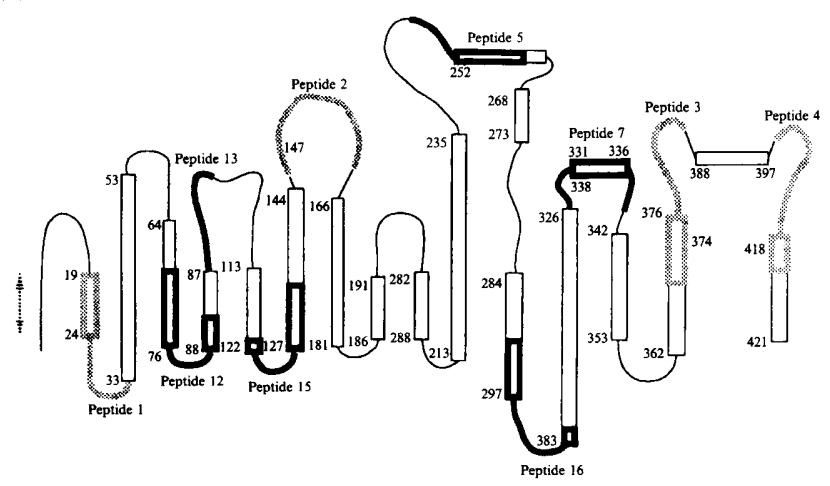

(b)

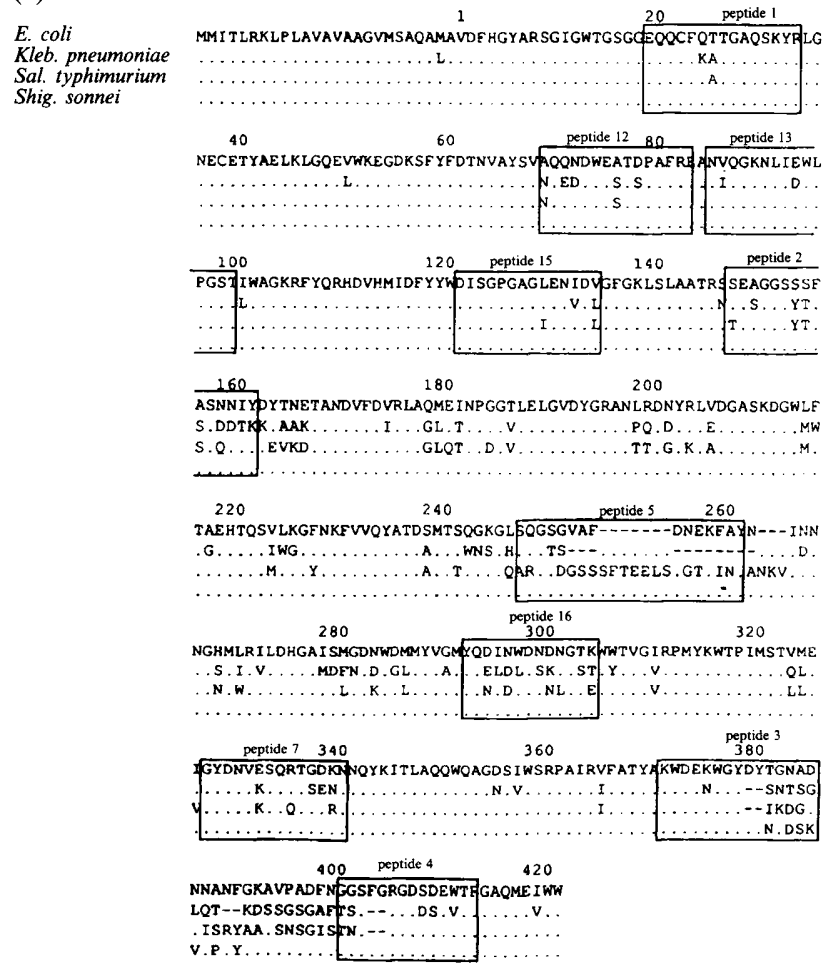

Fig. 1. (a) Position of peptides on the two-dimensional folding model of LamB (adapted from Charbit et al., 1991). Previously described peptides are stippled, new ones are black. Boxed residues represent $\beta$ strands, simple lines represent either turns or non-ordered regions of LamB. The approximate extent of the transmembrane region is represented by the arrow on the left. (b) Alignment of the four known LamB amino acid sequences. Positions of peptides are boxed. 


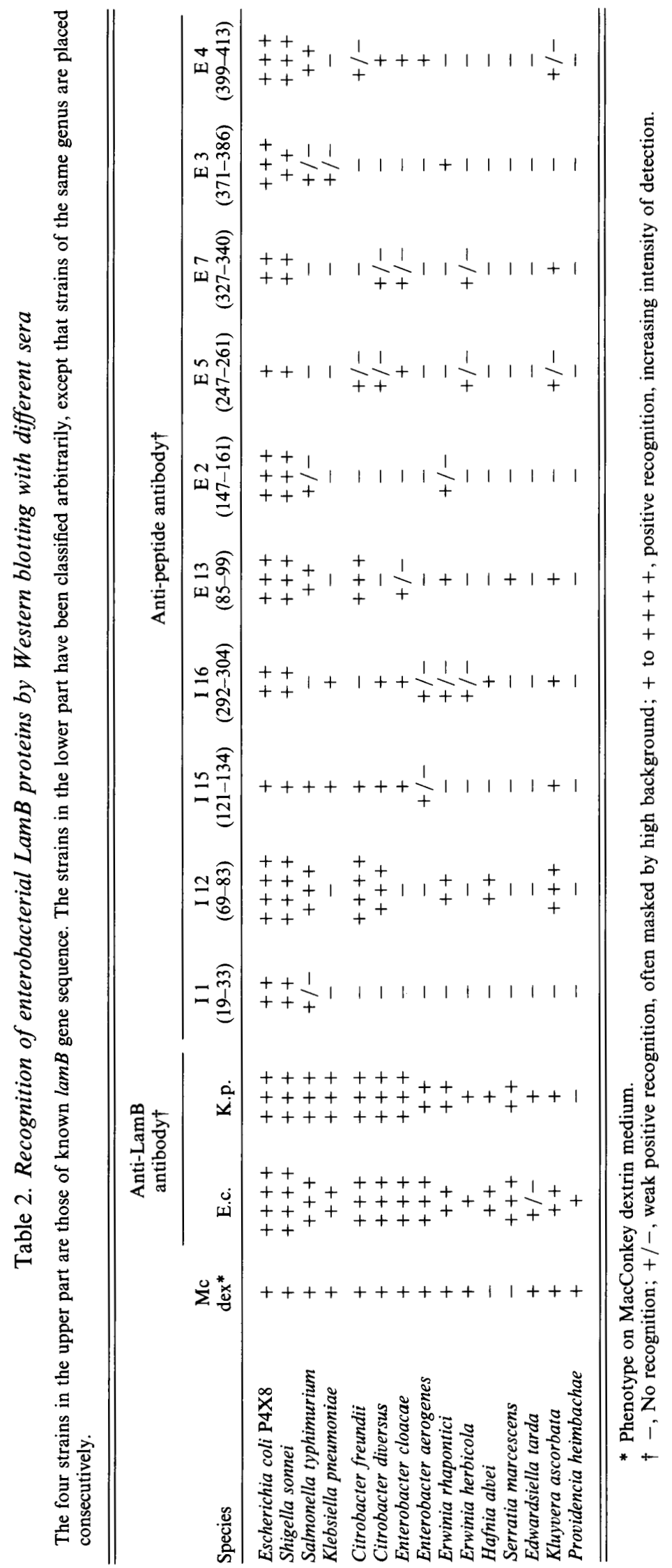


All the anti-peptide antibodies were able to react specifically with LamBE.c. in Western-blots with an extract of the LamB-positive $E$. coli strain ACl (Boulain et al., 1986), at a dilution of 1/3000. There was no detection with the LamB-negative strain pop6510.

Immunodetection experiments. Bacteria were grown at $30^{\circ} \mathrm{C}$ in liquid minimal medium supplemented with casamino acids (0.02\%; Difco), and maltose $(0.02 \%)$ as carbon source. At $\mathrm{OD}_{600}=1$, cells were harvested by centrifugation and concentrated 25 -fold in water. Samples were then collected, heat-denatured for $5 \mathrm{~min}$ at $100^{\circ} \mathrm{C}$ in loading buffer and run on 10\% SDS-polyacrylamide gels (approximately $5 \times 10^{7}$ bacteria per well). After electrophoretic transfer to a nitrocellulose filter, the extracts were analysed with the different antiLamB sera. In the Western blotting experiments, the anti-peptide sera were used at a final dilution of $1 / 1000$ (except anti-I 12, at $1 / 30000$ ) and the polyclonal anti-LamBE.c. and anti-LamBK.p. at 1/10000 and $1 / 4000$, respectively.

\section{Results}

The presence of a protein immunologically related to the E. coli $\mathrm{K} 12 \mathrm{LamB}$ protein was assayed by Western blotting on total cellular extracts from 15 enterobacterial strains with anti-LamBE.c. or anti-LamBK.p. antibodies raised against the denatured proteins (see Methods). It was also tested with six anti-LamBE.c. peptide antibodies directed against regions predicted to be at least partly cell-surface-exposed, and four antibodies directed against regions predicted to face, at least partly, the periplasm (Fig $1 a$ ). These 15 strains were chosen because they were all able to utilize maltose as carbon source (see Methods).

\section{Polyclonal antibodies directed against LamBE.c. and LamBK.p. proteins}

In all the members of the family Enterobacteriaceae studied, a maltose-inducible protein with an apparent molecular mass comparable to that of LamBE.c. (approximately $47 \mathrm{kDa}$ ) was detected by both antiLamBE.c. and anti-LamBK.p. antibodies (Fig. 2a,b). Providencia heimbachae was the only strain for which there was a weak recognition by anti-LamBE.c. antibody, and where specific recognition by anti-LamBK.p. antibody could not be ascertained because of important non-specific cross-reactions with other bacterial components. A good overall correlation was observed between the recognition patterns obtained with the two antibodies, indicating that the immunogenic epitopes of these two LamB proteins were at least partly conserved in different enterobacteria.

\section{Anti-peptide antibodies directed against internal regions of LamBE.c.}

Anti-I 1 antibody [I 1: amino acids (aa) 19-33 of LamBE.c.] recognized equally the $E$. coli and the Shigella sonnei (LamBS.s.) LamB proteins, but only poorly LamB from Sal. typhimurium (LamBS.t.), and not at all LamB from Kleb. pneumoniae (LamBK.p.). Changes were found in the sequence of this region in LamBS.t.) and LamBK.p., but not in LamBS.s. (Fig. 1), suggesting that the anti-I 1 recognition site lies within this central part of peptide I 1 . In all the other enterobacteria, no LamB-like protein was recognized by anti-I 1 antibody, indicating that region $I 1$ was subject to great antigenic polymorphism.

Anti-I 12 antibody (I 12: aa 69-83 of LamBE.c.) recognized LamBE.c., LamBS.s. and LamBS.t., but not LamBK.p. Two changes are found in this region in LamBS.t. (at the $\mathrm{N}$ terminus and in the middle of peptide 12). Since these changes did not affect detection by antiI 12, the recognition site of this antibody is probably located toward the C-terminal end of peptide 12 . This assumption is supported by the fact that the same two changes are present also in LamBK.p. The lack of recognition of LamBK.p. is likely to be due to the three additional changes found in the distal part of the peptide at positions 71, 72 and 78. Anti-I 12 antibody also recognized a LamB protein in the two members of the genus Citrobacter, Kluyvera ascorbata (a clinical isolate which is classified between Citrobacter and Enterobacter; Brenner, 1978), Hafnia alvei and Erwinia rhapontici (Fig. $2 b$ ).

Anti-I 15 antibody (I 15: aa 121-134 of LamBE.c.) recognized LamBE.c., LamBS.s., LamBS.t. and LamBK.p. proteins. It also recognized LamB from the two Citrobacter strains, Kluy. ascorbata and Enterobacter.

Anti-I 16 antibody (I 16: aa 292-304 of LamBE.c.) recognized LamBE.c. and LamBS.s., LamBK.p. poorly, but not LamBS.t. There are five changes in this region of LamBS.t. Anti-I 16 antibody also reacted with LamB from Kluy. ascorbata, C. diversus, Ent. cloacae and poorly with Ent. Aerogenes, Erw. rhapontici and Erw. herbicola.

Anti-peptide antibodies directed against external regions of LamBE.c.

Anti-E 13 antibody (E 13: aa 85-99 of LamBE.c.) recognized the LamB proteins from E. coli, Shig. sonnei and Sal. typhimurium, but not from Kleb. pneumoniae. The two changes in this region of LamBK.p. are probably responsible for the loss of recognition. Anti-E 13 antibody also showed specific cross-reaction with $C$. freundii, Ent. cloacae, Kluy. ascorbata and Serratia marcescens.

Anti-E 2 antibody (E 2: aa147-161 of LamBE.c.) recognized LamBE.c. and LamBS.s., LamBS.t. weakly, but not LamBK.p. This region is highly variable in LamBS.t. (five changes out of 15 residues) and LamBK.p. 


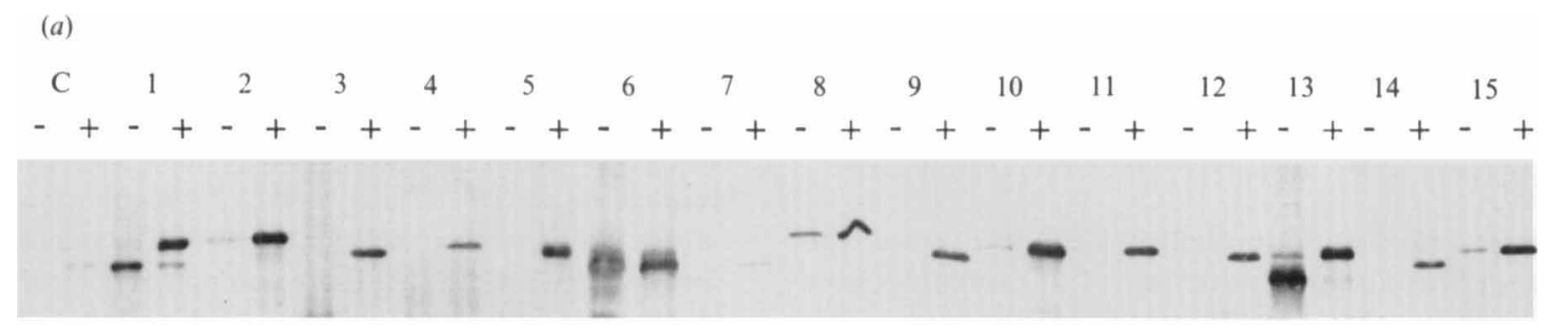

(b)

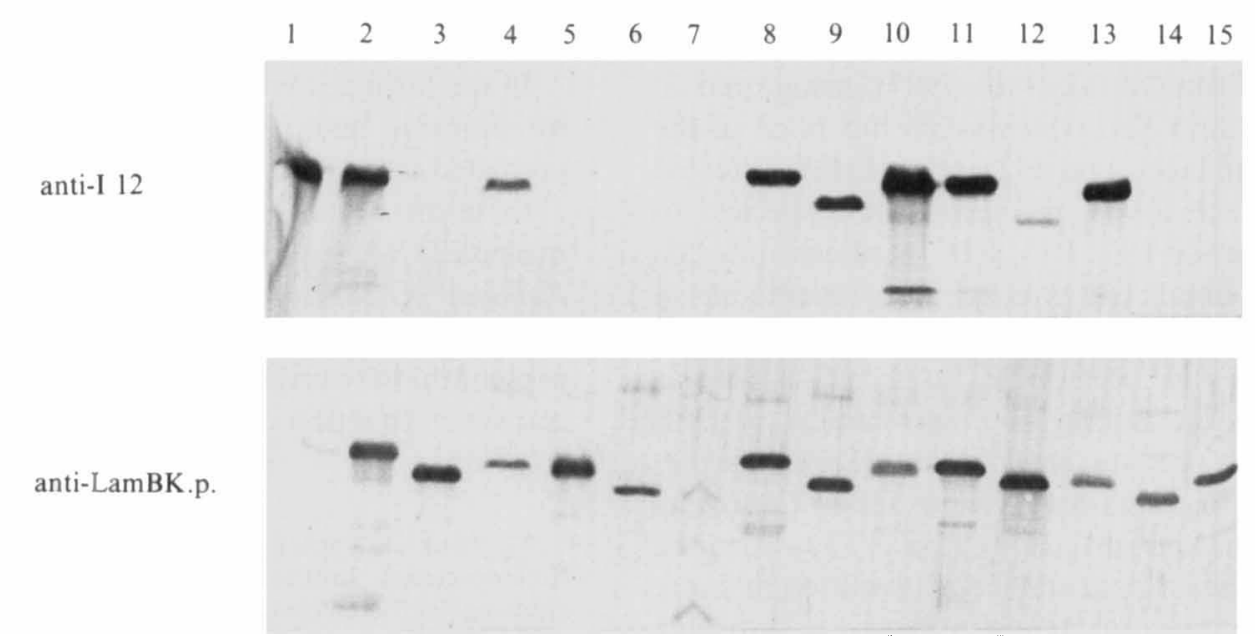

Fig. 2. Recognition of enterobacterial LamB proteins by Western blotting with different sera. (a) Recognition of LamB-like proteins in extracts from maltose-induced $(+)$ and non-induced $(-)$ enterobacterial cultures by anti-LamBE.c. serum. $(b)$ Recognition of LamB-like protein in extracts from maltose-induced enterobacterial cultures by anti-I 12 (upper part) or by anti-LamBK.p. (lower part) antibodies. The negative control $E$. coli pop6510 is not shown. To avoid the diffuse smear seen in $E$. coli P4X8 recognized by anti-I 12, the extract was diluted tenfold for the blot labelled with anti-LamBK.p. C, E. coli pop6510; 1, E. coli P4X8; 2, Sal. typhimurium; 3, Serr. marcescens; 4, H. alvei; 5, Kleb. pneumoniae; 6, Edw. tarda; 7, P. heimbachae; 8, Kluy. ascorbata; 9, Erw. rhapontici; 10, C. freundii; 11, C. diversus; 12, Ent. cloacae; 13, Shig. sonnei; 14, Erw. herbicola; 15, Ent. aerogenes.

(eight changes, with three changes identical to those present in LamBS.t.) (Fig. 1). Anti-E 2 did not react with any of the 12 other enterobacterial species (except weakly with Erw. rhapontici).

In addition to $E$. coli and Shig. sonnei, anti-E 5 antibody (E 5: aa 247-261 of LamBE.c.) reacted with Ent. cloacae and weakly with the two Citrobacter species, Erw. herbicola and Kluy. ascorbata. This antibody did not recognize LamBS.t. and LamBK.p. either, for which many changes (substitutions, deletions and insertions) were found in this region.

Anti-E 7 antibody (E 7: aa 327-340 of LamBE.c.) was able to recognize LamBE.c. and LamBS.s., but not LamBS.t. and LamBK.p., for which three and four changes were found in this region, respectively. Anti-E 7 reacted positively with Kluy. ascorbata, and only weakly with $C$. diversus, Erw. herbicola, and Ent. cloacae.

Anti-E 3 antibody (E 3: 371-386 of LamBE.c.) recognized LamBS.s. well, and LamBS.t. and LamBK.p. poorly. Among the 11 other enterobacteria tested, antiE 3 antibody reacted only with Erw. rhapontici.

Anti-E 4 antibody (E 4: aa 399-413 of LamBE.c.) recognized LamBE.c., LamBS.s. and LamBS.t., but not LamBK.p. Comparison of LamBS.t. and LamBK.p. amino acid sequences suggest that the anti-E 4 antibody recognition site (which was previously shown to be cellsurface-exposed; Molla et al., 1987) lies within the distal part of peptide E 4. Anti-E 4 antibody reacted also with the two Enterobacter species and $C$. diversus (and only very poorly with $C$. freundii and Kluy. ascorbata).

\section{Discussion}

A maltose-inducible protein, immunologically related to the $E$. coli maltoporin was detected by an anti-LamBE.c. serum in all the $\mathrm{Mal}^{+}$enterobacteria tested. Proteins in all but one were also recognized by an anti-LamBK.p. serum. However, recognition of the various proteins by anti-peptide sera raised against 10 loop regions of LamBE.c. was very variable, showing that these regions of the protein present high antigenic variability.

The regions of peptides E 2, E 5, E 3 and E 4, have been demonstrated previously to be cell-surface-exposed (Molla et al., 1987; Charbit et al., 1991). Amino acid 
comparison of the four $\mathrm{LamB}$ proteins sequenced showed that these four regions were highly variable (Werts et al., 1992; Fig. 1b). Our results showed that these regions were also antigenetically highly polymorphic.

There was no correlation between the location of the peptides chosen for the induction of anti-peptide antibodies, within the LamB protein (cell surface or periplasmic), and the degree of conservation of the antigenic determinants. For example anti-peptide I 1, directed against a region of LamBE.c. previously shown to face the periplasm (Molla et al., 1987), recognized $E$. coli, Shig. sonnei and Sal. typhimurium but none of the other species. Similarly, anti-E 2 and anti-E 3, directed against cell surface loops, recognized few species. In contrast, anti-peptide I 12, I 15, I 16 (predicted internal loops) and E 13 (predicted external loops) were able to recognize a LamB protein in 8 to 10 of the 15 enterobacteria tested. Region E 7 (aa 327-340) was antigenically variable among the enterobacteria. This is in agreement with previous genetic data (Desaymard et al., 1986) which suggested that residue 333 of LamBE.c. was exposed at the cell surface. Region E 13 toward the $\mathrm{N}$ terminus of the protein (aa 85-99), although more conserved, also appeared to be subject to antigenic variability, which suggests that it is exposed on the surface of the protein.

The LamB proteins from three strains ( $P$. heimbachae, Edwardsiella tarda and Serr. marcescens) were only poorly or not recognized by the anti-peptide sera. In addition, the LamB proteins of $P$. heimbachae and $E d w$. tarda were only poorly or not recognized by the antiLamBE.c. or anti-LamBK.p. antibodies. Interestingly, these two strains could still use dextrins as carbon source, suggesting that in spite of important antigenic variability in the surface loops of the protein, the function and thus the overall structure might be conserved. Similar results were observed with the ferric enterobactin receptor FepA, an outermembrane protein for which it was found that distantly related bacteria contained an outermembrane protein that transported the native $E$. coli siderophore but possessed only slight structural similarity to E. coli FepA (Rutz et al., 1991). The other strain (Serr. marcescens was unable to use dextrins as carbon source (Table 1 and Methods). However, it possessed a protein still immunologically related to LamBE.c., since it was recognized by both the anti-LamBE.c. and anti-LamBK.p. sera. Thus, in spite of the loss of the two main known functions of LamBE.c. (phage reception and dextrin utilization), this protein still shares antigenic determinants with LamBE.c.

The Shig. sonnei LamB protein is the most related to the $E$. coli $\mathrm{K} 12 \mathrm{LamB}$ protein. It was the only protein to be recognized by all the sera, and the only one to have conserved the phage receptor function of LamB from $E$. coli K12 (see Methods). None of the other bacterial species were sensitive to any of the LamB-specific phages tested (data not shown). Other than the species least related to $E$. coli ( $E d w$. tarda, $P$. heimbachae and $H$. alvei), which were either poorly or not recognized by the anti-peptide antibodies, there was no correlation between the pattern of antibody recognition and the phylogenic relationships between the strains tested. In fact, closely related strains (for example $C$. freundii and $C$. diversus) often had different recognition patterns.

In the future, it will be interesting to extend this study by choosing new regions of the protein, in particular predicted conserved regions.

In terms of the topology and folding of the LamB protein, DNA sequence analysis of the $\operatorname{lam} B$ genes from $P$. heimbachae, and Edw. tarda, should provide particularly interesting information on the regions of the protein that are critical for the conservation of the sugar transport function of LamB, and thus for its overall structure.

We thank Dr Patrick Grimont and Elisabeth Ageron (Unité des Entérobactéries, Institut Pasteur, Paris) for kindly providing and checking the enterobacterial strains. This work was supported by grants from the Association pour le développement de la Recherche sur le cancer, the Ligue Nationale contre le Cancer, the Fondation pour la Recherche Médicale and the North Atlantic Treaty Organization (NATO).

\section{References}

Bloch, M. A. \& Desaymard, C. (1985). Antigenic polymorphism of the LamB protein among members of the family Enterobacteriaceae. Journal of Bacteriology 163, 106-110.

Boulain, J. C., Charbit, A. \& Hofnung, M. (1986). Mutagenesis by random linker insertion into the lamB gene of $E$. coli K12. Molecular and General Genetics 205, 339-348.

BRENNER, D. J. (1978). Characterization and clinical identification of enterobacteriaceae by DNA hybridization. Progress in Clinical Pathology VII, 71-117.

Charbit, A. \& Hofnung, M. (1985). Isolation of different bacteriophages using the LamB protein for adsorption on $E$. coli K12. Journal of Virology 53, 667-671.

Charbit, A., Gehring, K., Nikaido, H., Ferenci, T. \& Hofnung, M. (1988). Maltose transport and starch binding in phage resistant point mutants of maltoporin: functional and topological implications. Journal of Molecular Biology 201, 487-496.

Charbit, A., Ronco, J., Michel, V., Werts, C. \& Hofnung, M. (1991). Permissive sites and the topology of an outer-membrane protein with a reporter epitope. JOurnal of Bacteriology 173, 262-275.

Clément, J. M. \& Hofnung, M. (1981). Gene sequence of the lambda receptor, an outer membrane protein of $E$. coli K12. Cell 27, 507-514.

Desaymard, C., Debarbouille, M., Jolit, M. \& Schwartz, M. (1986). Mutations affecting antigenic determinants on an outer membrane protein of Escherichia coli. EMBO Journal 5, 1383-1388.

Francoz, E., Molla, A., Dassa, E., Saurin, W. \& Hofnung, M. (1990). The maltoporin of Salmonella typhimurium: sequence and folding model. Research in Microbiology 141, 1039-1059.

Hamel, J., Dugourd, D., Martin, D., Proulx, C., Chong, P. \& BrodeUR, B. R. (1992). Localization of conserved B-cell epitopes 
among encapsulated and non-encapsulated Haemophilus influenzae P2 porin proteins using synthetic peptides. Journal of General Microbiology 138, 161-168.

Harlow, E. \& Lane, D. (1988). Antibodies. A Laboratory Manual. Cold Spring Harbor, NY Cold Spring Harbor Laboratory.

HengGe, R. \& Boos, W. (1983). Maltose and lactose transport in $E$. coli. Examples of two different types of concentrative transport systems. Biochimica et Biophysica Acta 737, 443-448.

MerRIFIELD, R. B. (1963). Solid phase peptide synthesis. I. The synthesis of a tetrapeptide. Science 85, 2149-2154.

Molla, A., Charbit, A., Le Guern, A., Ryter, A. \& Hofnung, M. (1989). Antibodies against synthetic peptides and the topology of LamB, an outer-membrane protein from Escherichia coli K12. Biochemistry 28, 8234-8241.

NiKaido, H. \& VAaRA, M. (1985). Molecular basis of bacterial outer membrane permeability. Microbiological Reviews 49, 1-32.

PICK, K. H. \& WOBER, G. (1979). Maltodextrin pore proteins in the outer-membrane of Escherichia coli and Klebsiella pneumoniae: Immunological comparisons. FEMS Microbiology Letters 5, 119122.

Roessner, C. A. \& Ihler, G. M. (1987). Sequence of amino acids in LamB responsible for spontaneous ejection of bacteriophage lambda DNA. Journal of Molecular Biology 195, 963-966.

Rutz, J. M., Abdullah, T., Singh, S. P., Kalve, V. I. \& Klebba, P. E. (1991). Evolution of the ferric enterobactin receptor in Gramnegative bacteria. Journal of Bacteriology 173, 5964-5974.

Singh, S. P., Upshaw, Y., Abdullah, T., Singh, R. S. \& Klebba, P. E. (1992). Structural relatedness of enteric bacterial porins assessed with monoclonal antibodies to Salmonella typhimurium $\mathrm{OmpD}$ and OmpC. Journal of Bacteriology 174, 1965-1973.

Werts, C., Charbit, A., Bachellier, S. . \& Hofnung, M. (1992). DNA sequence analysis of the $\operatorname{lam} B$ gene from $K$. pneumoniae. Implications for the topology and the pore functions in maltoporin. Molecular and General Genetics 233, 372-378. 